\title{
Multidimensional Heterogeneous Medical Data Push in Intelligent Cloud Collaborative Management System
}

\author{
Gang Liu' ${ }^{1}$ and Xiaofeng $\mathrm{Li} \mathbb{D}^{2}$ \\ ${ }^{1}$ College of Computer Science and Technology, Harbin Engineering University, Harbin 150001, China \\ ${ }^{2}$ Department of Information Engineering, Heilongjiang International University, Harbin 150025, China
}

Correspondence should be addressed to Xiaofeng Li; lixiaofeng@hiu.net.cn

Received 5 July 2020; Revised 3 October 2020; Accepted 7 October 2020; Published 19 October 2020

Academic Editor: Abd E. I.-Baset Hassanien

Copyright (c) 2020 Gang Liu and Xiaofeng Li. This is an open access article distributed under the Creative Commons Attribution License, which permits unrestricted use, distribution, and reproduction in any medium, provided the original work is properly cited.

\begin{abstract}
The medical data in the intelligent cloud collaborative management system have multidimensional heterogeneous interference, and there are problems such as low data information update rate and poor push results in the push process. Therefore, a method for multidimensional heterogeneous medical data push was proposed. First of all, the logical architecture of the multidimensional heterogeneous data push system was determined, and the data push function was designed; secondly, redundant data removal and noise reduction preprocessing were conducted against the push data, correlation rules were used to integrate multidimensional heterogeneous medical data, the weight of medical data was calculated, the heterogeneous data matrix was constructed, and the integrated medical data were weighted to eliminate multidimensional heterogeneous interference. The results show that the data update rate of the proposed method is faster, the user retention and communication rate are high, the data push precision rate is over $80 \%$, and the recall rate is as high as $76 \%$. Therefore, its performance is significantly better than traditional methods.
\end{abstract}

\section{Introduction}

Multidimensional medical data refer to medical data with a multidimensional structure defined by dimensions and measures, which is the main object of data online analysis. In recent years, with the development of hospital informatization construction, computer information technology and database management technology in particular have been widely used in the medical field, and a large number of medical data with different dimensions and forms have emerged [1]. As a result, the single data management platform can no longer satisfy the medical data processing requirements [2]. In the information age, cloud computing technology shows obvious advantages and rapid development. To this end, an intelligent cloud collaborative management system with collaborative processing function has been developed for the purpose of coordinating and managing multidimensional heterogeneous medical data, and integrating medical information, and effectively enhancing the application effectiveness of medical data $[3,4]$. The main principle of the design and implementation of an intelligent cloud collaborative management system is to combine the task collaboration system and information management system through artificial intelligence technology under the premise of no mutual influence. Therefore, the intelligent cloud collaborative management system can perform work planning tasks and information management tasks, respectively. For hospital management, high-quality data makes management efficiency more effective and more precise $[5,6]$. In the management decision-making on medical data, it is usually impossible to quickly extract key data from hospital big data and drive smart healthcare with data, but only through statistical reports submitted by the Statistics Division and Information Division as well as statistical reports in various discrete systems for management decisions [7, 8]; in this case, the intelligent cloud collaborative management system can be analyzed by multidimensional medical data push.

The mass medical information feature push method based on data feature matrix obtained the medical data 
feature matrix array by using the medical big data feature intelligent collection method $[9,10]$, matched all patient group information and part of the patient information by using the data feature matrix array, integrated the corresponding patients into the patient group with the highest similarity, and matched the keywords in the similar patient group and the basic key data features pushed in the medical data feature matrix array, so that the patient group where the patients were located would push medical messages according to the priority push method of location-based service (LBS) [11]. Some scholars analyzed the feasibility of the application of information push technology in the management of tuberculosis patients in the floating population and provided a reference for changing the management model. They used self-made questionnaires to investigate the basic conditions of patients, treatment compliance, personal communication tools, and other information. The conclusion shows that the information push technology is feasible to manage tuberculosis patients in the floating population, and patient acceptance is high [12]. Therefore, the application of information push technology has good feasibility in the management of migrating tuberculosis patients and high patient acceptance. In the proposed method, the Internet-based inpatient health education cloud platform was used to push and set up sharing; that is, based on the characteristics of hospitals and departments $[13,14]$, this method proposed specific nursing units, regularly pushed propaganda and education information and required its sharing rate to be greater than $80 \%$, regularly shared the feedback information and conclusions, and analyzed the application effect of health propaganda and education cloud platform for Internet-based inpatients according to the patient satisfaction questionnaire, the reading amount of propaganda and education content and the opinions of the patients visited [15].

The above-mentioned traditional management systems can achieve better management functions, and they are widely used in actual work and life. However, according to the studies on their long-term applications, the traditional systems have problems such as long query time and inability to update system information data in time. Therefore, it is necessary to introduce data push technology to improve the updated function of the system. In this system optimization, multidimensional heterogeneous medical data push technology is applied. Among them, "multidimensional" mainly refers to the dimension of data to be pushed, that is, the number of independent parameters in mathematics; "heterogeneous" is a parameter that contains different components and properties. Multidimensional heterogeneous data is the push data message that is not unique in dimension and has a different network structure. Through the application of multidimensional heterogeneous medical data push technology, the function of data push can be realized, thereby solving the problems of long query time and system information data cannot be updated in time in the traditional system and improving the query ability and update speed of the system.

The main contributions of this study are as follows.

(1) The multidimensional heterogeneous medical data push technology is applied to intelligent cloud collaborative management system for analysis; (2) the logical architecture of the multidimensional heterogeneous data push system is determined, which lays the foundation for subsequent research; (3) weighted analysis is made on multidimensional heterogeneous medical data to eliminate multidimensional heterogeneous interference, which provides a basis for improving the efficiency of medical data push; (4) the push channel is selected, which greatly improving the effect of data push.

\section{Related Work}

By deeply analyzing the Browser/Server (B/S) architecture, Bo [16] designed the corresponding function modules according to the system design principles and equipped with a reasonable database to ensure the quick connection of database; the test results show that the designed electronic medical record management system could quickly enter and query medical record data, achieve functions such as reliable storage of medical record data, and have an important auxiliary role for the hospital to grasp a medical record in time. Jin et al. [17] proposed a wireless intelligent collaboration system based on generalized spatial modulationmedia based modulation (GSM-MBM), which could activate multiple antennas at the relay and install radio frequency mirrors near the antennas; different channel paths were constructed by activating different radio frequency mirrors, so as to carry extra information bits; the system transmission efficiency, average paired error probability, and energy consumption gain were deducted according to relevant theories, and Monte Carlo simulations were conducted; as a result, the transmission efficiency was improved, and the bit error rate and energy consumption were reduced; however, the bit error rate was slightly higher under the same transmission efficiency, yet the required the number of required transmitting antennas was greatly reduced, thereby reducing the complexity and cost of system implementation. Aiming at the data characteristics of space structure health monitoring, Zhang et al. [18] put forward the overall framework of the space structure health monitoring Internet of Things (IoT) system and established the application layer data processing algorithm by taking the advantages of cloud computing in processing intensive tasks, completed the design of the cloud data management system for spatial structure monitoring, and conducted real-time processing and interactive display over the monitoring information of multiple large-span spatial structures including National Stadium and Hangzhou Railway Station. Qin et al. [19] designed a medical imaging remote diagnosis cloud service platform to realize automatic uploading, centralized storage and management of image data of primary medical institutions, as well as the sharing of image information and diagnosis reports between hospitals; the system construction and research on the image cloud platform were carried out from the perspectives of registration of image data, the design of data storage center and access to image. Liu et al. [20] proposed a fine-grained access control (FGUR) solution that supported user revocation, which by introducing the attribute hierarchy into the Comparison-Based Encryption 
(CBE) and combined with the Broadcast Ciphertext-Policy Attribute-Based Encryption (BCP-ABE), efficiently implemented fine-grained access control and real-time user revocation in the personal health record (PHR) cloud management system; compared with CBE, the FGUR solution shows better performance in encryption overhead and dynamic access permissions.

\section{Design of a Collaborative Management System for Multidimensional Heterogeneous Medical Data Push}

The multidimensional heterogeneous medical data push technology is the core technology applied in the intelligent cloud collaborative management system. Therefore, the implementation environment and function execution program of multidimensional heterogeneous medical data push technology is introduced for system design.

3.1. Logical Architecture of the System. In the application of multidimensional heterogeneous medical data push technology to the intelligent cloud collaborative management system [21, 22], the server actively sends messages to the receiver, and the system user does not need to actively check and update; the system can push all multidimensional heterogeneous medical data to users via the intelligent cloud server system, the system users can receive the most recent medical data information [23]. Therefore, the logical architecture of the Intelligent Cloud collaboration management system is shown in Figure 1.

According to Figure 1, the logical architecture of the intelligent cloud collaborative management system is mainly composed of a cloud data layer, data management layer, application interface layer, and access layer. Among them, the cloud data layer is to integrate the multidimensional medical data into a data set after receiving the user-level access information. The data management layer is to process the integrated data set to realize the collaborative work of data and push [24, 25]; the application interface layer and access layer are mainly aimed at the receiver, the system can push the required diversified and heterogeneous medical data according to the setting requirements of the user terminal, so as to realize the collaborative work of data and push [26].

3.2. Design of Data Push Function. In the functional design of the collaborative management system software using multidimensional heterogeneous medical data push, this method allows the system to realize the function of medical data push based on the traditional collaborative management function, and tries not to interfere or affect the original collaborative management function during the operation of the new function. Therefore, the push function of multidimensional heterogeneous medical data was specifically designed in this study. It is shown in Figure 2.

According to Figure 2, the heterogeneous data information was mostly collected, and the data was transmitted to the user terminal through the access request; the multidimensional heterogeneous information was collected into the information database, and the weights and push decisions were defined through the access request and then transmitted to the user terminal. After the users successfully subscribed to the content of the cloud push platform, the platform needed to send messages to its own users and push messages to the client in real-time through the long connection established between the cloud and the client $[27,28]$. Based on traditional push, the proposed cloud push process was carried out in the cycle of "Subscription-Collection-Decision-Push." The cloud push cycle is shown in Figure 3.

3.3. Cross-Layer Preprocessing of Push Data. In the use of heterogeneous sensors to collect and store original data in the database, this study selects part of the multidimensional heterogeneous medical data in the database as the original information push data. Before the medical data was pushed, cross-layer preprocessing was first performed to reduce the error rate of the data push and to improve the data push quality $[29,30]$. The entire data cross-layer preprocessing process is divided into two steps: the removal of redundant data and the noise reduction of data.

Assuming that the original data set is $n$, the data feature set is $m(f)$, and $f$ represents the eigenvalue, then the probability relationship between the original data set $n$ and the data feature set $m(f)$ can be expressed as follows:

$$
D_{\text {sensor }}(f)=n \int_{1}^{R} m(f) d(f),
$$

where $D_{\text {sensor }}(f)$ represents the eigenvalue probability of data set $n$; the solution result of equation (1) is the probability distribution function of the measured value of the medical data push, that is, the push data between the data layer frequency $[1, R]$. According to the solution results, $m$ and $n$ can be divided into three situations. It is shown in Figure 4.

When the solution result is situation 3 in Figure 4, the redundant data in the medical data set $n$ should be removed.

The noise reduction processing was conducted over data set; the frequency-based eigenvalue probability distribution in the multidimensional collaborative processing under the normal transmission link is shown in the following equation:

$$
F_{x}(f)=\iint_{v, \varphi} f(v, \varphi) \mathrm{d} v \mathrm{~d} \varphi, f(v, \varphi)=u\left(\sqrt{v^{2}+\varphi^{2}+\cos \left(\frac{1}{2} \pi+\omega\right)}\right),
$$




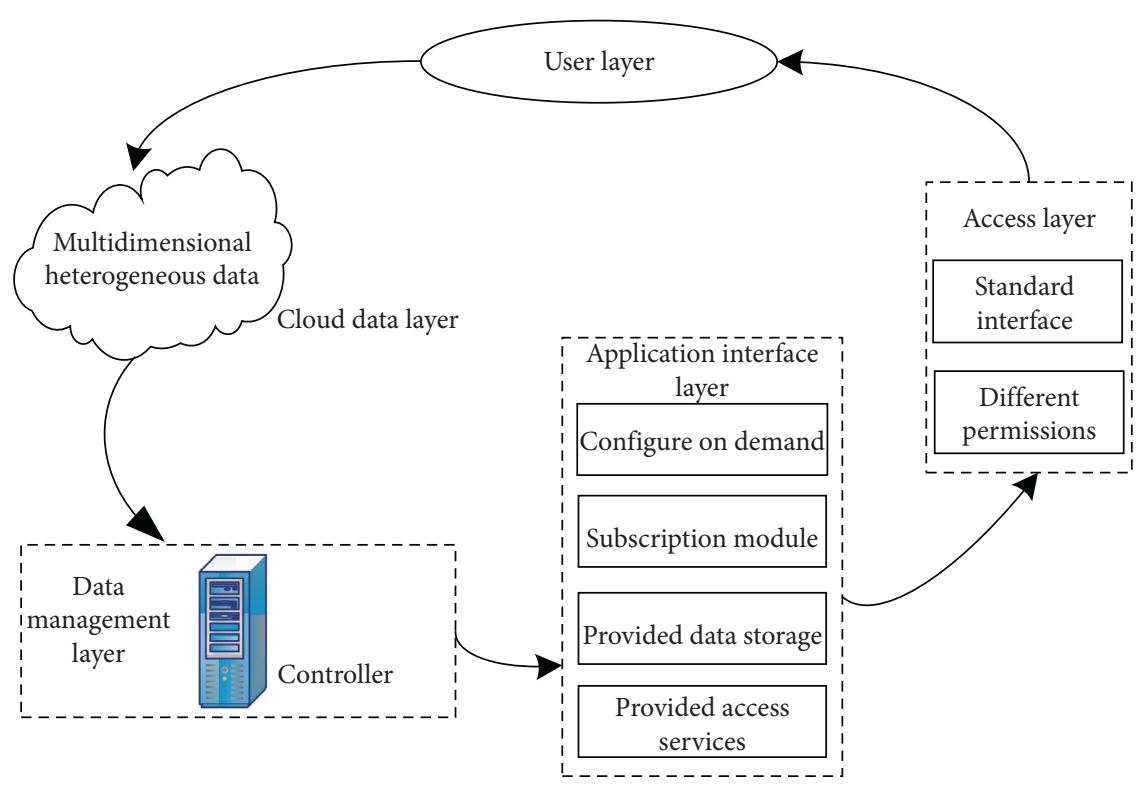

FIGURE 1: Logical architecture of intelligent cloud collaboration management system.

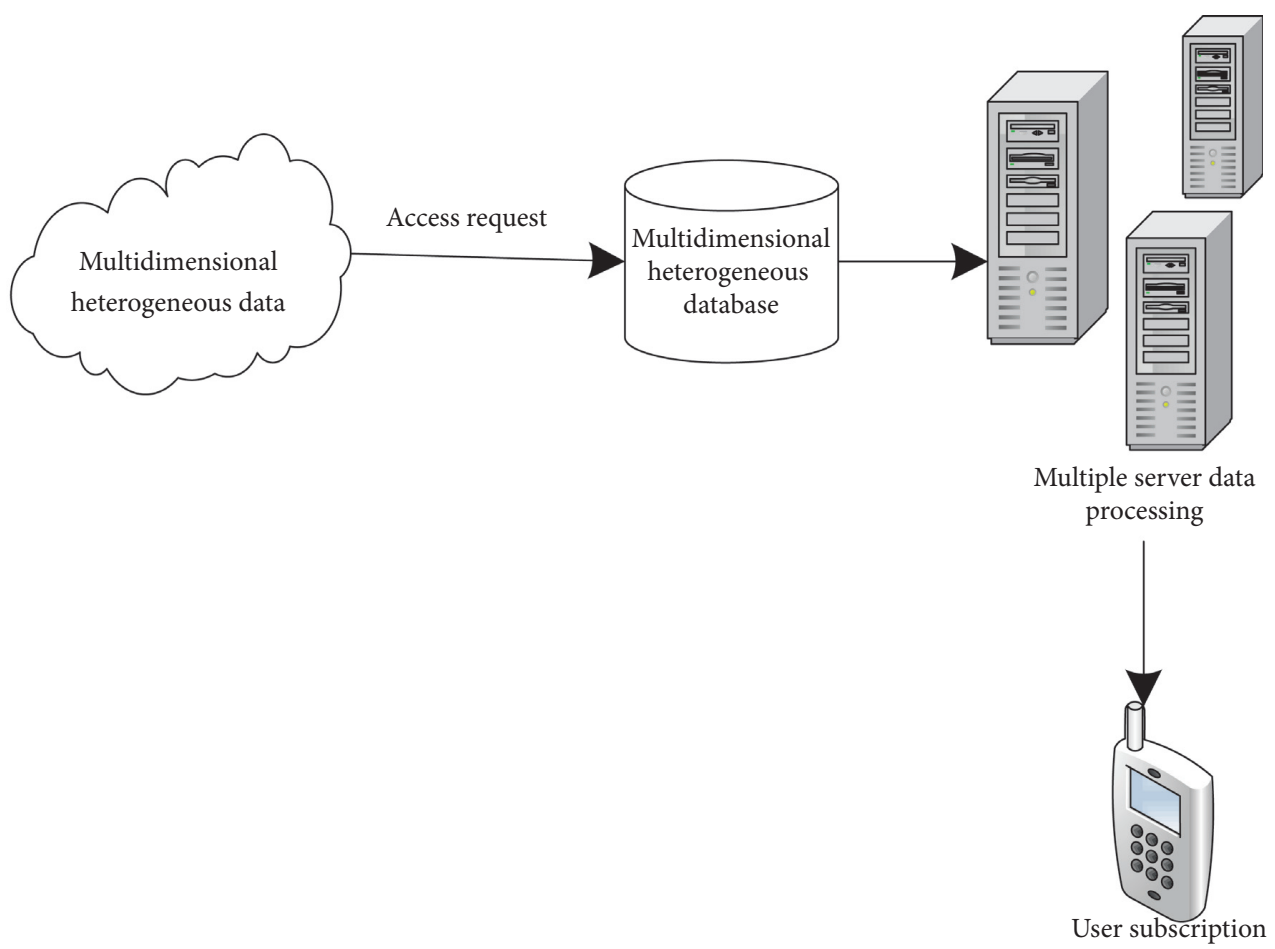

Figure 2: Design of data push function.

where $F_{x}(f)$ is the eigenvalue probability distribution function under the normal transmission link, and $f(v, \varphi)$ is the probability function. The parameters $v$ and $\phi$, respectively, represent the collaboration scale of the push node and the probability of maintaining the collaboration state, and the parameter $\omega$ is the angular frequency at which the heterogeneous sensor works.
Enhancement processing is performed on the effective signals in the medical push data, and the enhancement result $C$ is shown in the following equation:

$$
C=\left\{\begin{array}{l}
F_{x}(f) \frac{v \varphi}{2 \omega}, \\
2 v \varphi \cos \omega .
\end{array}\right.
$$




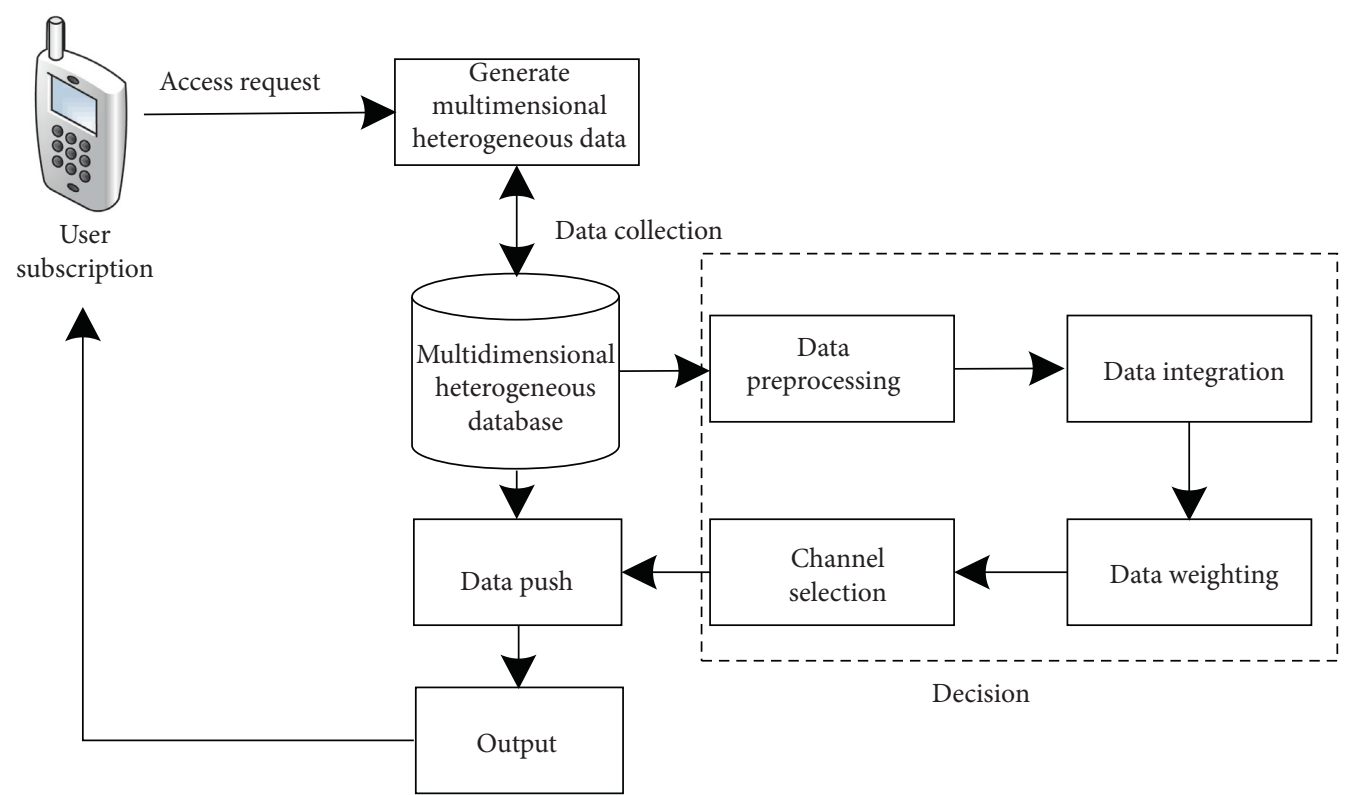

Figure 3: Cloud push cycle.

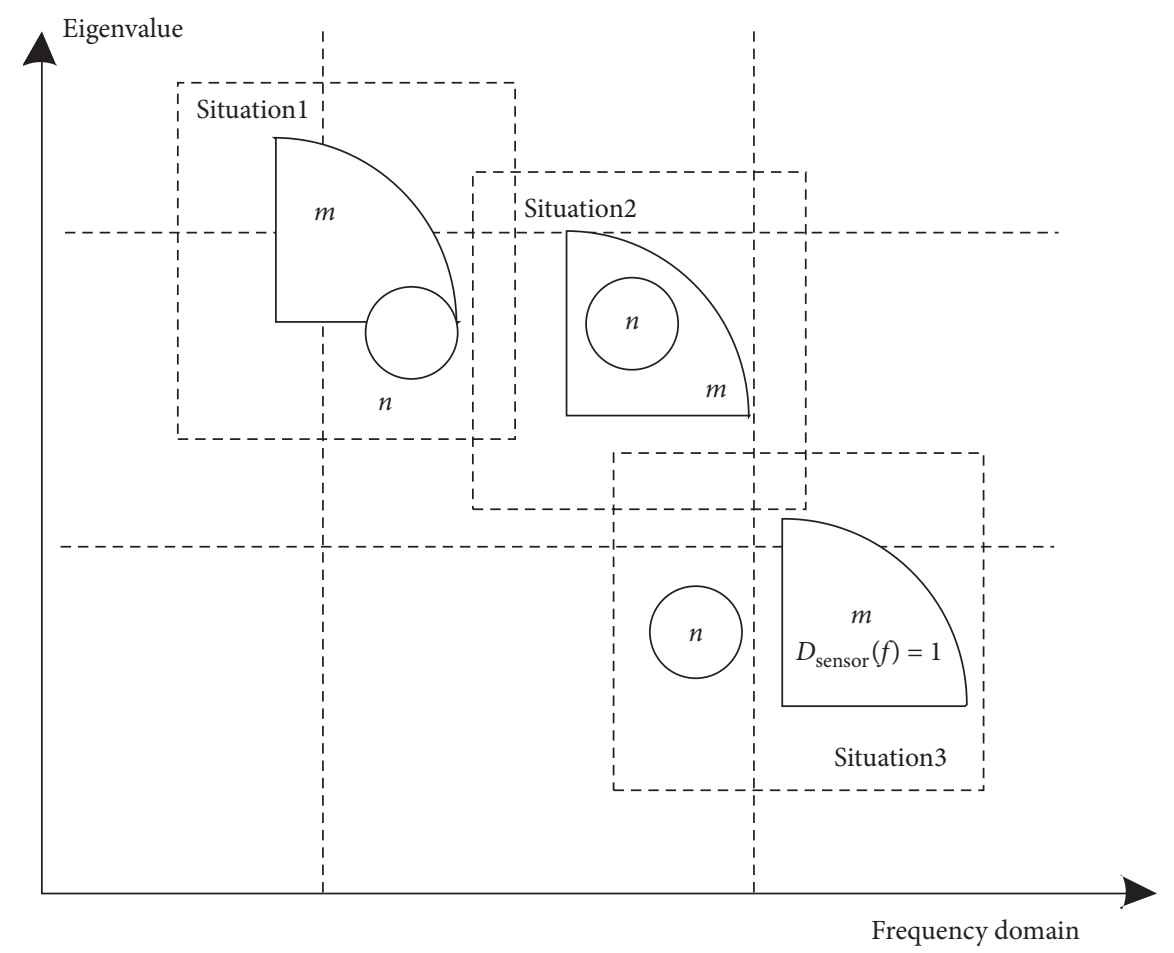

FigURE 4: Process of multidimensional heterogeneous data elimination and optimization.

According to equation (3), the strength of the effective data signal can be enhanced while reducing the noise signal $[31,32]$.

Combined with the above steps, the cross-layer preprocessing of push data is completed.

\subsection{Integration of Multidimensional Heterogeneous Medical} Data. The principle of integrated management of multidimensional heterogeneous medical data is that the correlation rules algorithm defines strong correlation rule parameters as minimum support and minimum confidence [33]. Among them, the support degree can be specifically defined according to the following equation:

$$
\operatorname{support}(A \longrightarrow B)=P(A \cup B) \text {. }
$$

Equation (4) is the probability that multidimensional heterogeneous medical data $A$ and data $B$ appear 
simultaneously. If the value of the calculation result is small, the correlation between $A$ and $B$ will be low. Similarly, the confidence in the correlation rule algorithm can be expressed as follows:

$$
\text { confidence }(A \longrightarrow B)=P(A \mid B) \text {. }
$$

Equation (5) is the probability of $B$ appearing when data $A$ appears. If the calculation result is $100 \%$, the correlation between $A$ and $B$ will be high. The correlation of any two data in the sample data can be obtained by synthesizing the two parameters. In addition, the integration results of multidimensional heterogeneous medical data are obtained after the correlated data is clustered and integrated.

3.5. Weighted Analysis of Integrated Medical Data. The analysis of multidimensional heterogeneous medical data is to analyze the content of medical information data. The analysis results can be used as a reference to eliminate multidimensional heterogeneous interference and help the system select an appropriate push method [34]. Besides, the weighted analysis of data was performed after the integration of medical data was completed.

First of all, the weight of the heterogeneous medical data in each dimension was calculated using the following equation:

$$
\mathrm{TF}(i)=\sum_{i=1}^{n} t f_{j}(i) \times \operatorname{class}(j)
$$

where $\operatorname{TF}(i)$ represents the weight of medical data, $t f(i)$ is the frequency of phrases appearing in a certain area in medical data, and class $(j)$ is the weight coefficient of regional evaluation, which can be obtained by the system controller [35]. The selected data were arranged in descending order of weight, and the component analysis of medical data was made. The component value is defined by $X_{i}$, then the main component value of each multidimensional heterogeneous medical data can be expressed as $X_{i}(i=1,2, \ldots, n)$ and filled into the following equation, so as to get the heterogeneous data matrix:

$$
S=\operatorname{TF}(i)\left[X_{1}, X_{2}, X_{3}, \ldots, X_{n}\right],
$$

where $S$ is heterogeneous data matrix. The transposed matrix of the matrix $S$ is obtained using the following equation:

$$
S^{T}=\operatorname{TF}(i)\left[\begin{array}{c}
X_{1} \\
X_{2} \\
X_{3} \\
\cdots \\
X_{n}
\end{array}\right],
$$

where $T$ represents the transposed symbol. Equations (4) and (5) are multiplied and the average value is obtained as $\operatorname{avg}_{X}$. Then, the overall heterogeneous value of the multidimensional heterogeneous medical data can be calculated using the following equation:

$$
V=S^{T} \sum\left\{X_{i}-\mu \| \operatorname{avg}_{X}-\left.\mu\right|^{T}\right\}
$$

where $N$ is the total amount of initial integrated medical data, and $\mu$ is the data offset parameter. If multidimensional heterogeneous medical data needs to be pushed simultaneously, the principal component of each data should be converted to $X_{i j}$; therefore, the conversion result of matrix $\mathrm{S}$ is shown in the following equation:

$$
S=\operatorname{TF}(i)\left[\begin{array}{lll}
X_{11} & X_{12} & X_{1 j} \\
X_{21} & X_{22} & X_{2 j} \\
& & \\
X_{i 1} & X_{i 1} & X_{i j}
\end{array}\right] .
$$

The calculation result of $V$ is then expressed in the form of matrix. Through the calculation of $\mathrm{V}$ and its diagonal matrix, the weighted calculation eigenvalue $W$ of the data can be solved. It is shown in the following equation:

$$
W=|V-k E|=S\left|V \cdot V^{\wedge}\right|
$$

where $E$ represents the identity matrix, and $V^{\wedge}$ parameter represents the diagonal matrix of $V$, from which the specific value of eigenvalue $k$ can be obtained. $k$ and $V$ together measure the heterogeneity of different medical data.

3.6. Selection of Data Push Channel. After the weighted analysis of integrated medical data, preparations were done to push information to the system. A push channel needed to be utilized in this process. Therefore, it was crucial to select the data push channel, which would directly affect the accuracy of push results.

The selection of data push channel should be specifically considered from two aspects: the carrying capacity of the push channel and the length of the push channel. First, the maximum efficiency of data transmission between a certain user $u$ and the push server $r$ should be calculated. It is shown in the following equation:

$$
U_{\mathrm{ur}}=\max _{r \in R}\left[\frac{c_{\mathrm{ur}}}{D_{\mathrm{ur}}}\right],
$$

where $c_{\text {ur }}$ represents the number of data transmissions used by user $u$, and $D_{\text {ur }}$ represents the total amount of data transmission required by user $u$. In addition, the matching value $\mathrm{MTM}_{\mathrm{ur}}$ of the maximum task medical data needs to be calculated:

$$
\mathrm{MTM}_{\mathrm{ur}}=U_{\mathrm{ur}} \varphi,
$$

where $\varphi$ is the matching parameter. All the channels that meet the requirements of the above equations are taken as candidate channels, and the channels are arranged according to the transmission distance of the channels. The specific arrangement is shown in Figure 5.

In Figure 5, the signaling channel controls the connection of channels and transfers network management information; physical channel reconfiguration can be used to achieve cofrequency hard handover and compression. The 

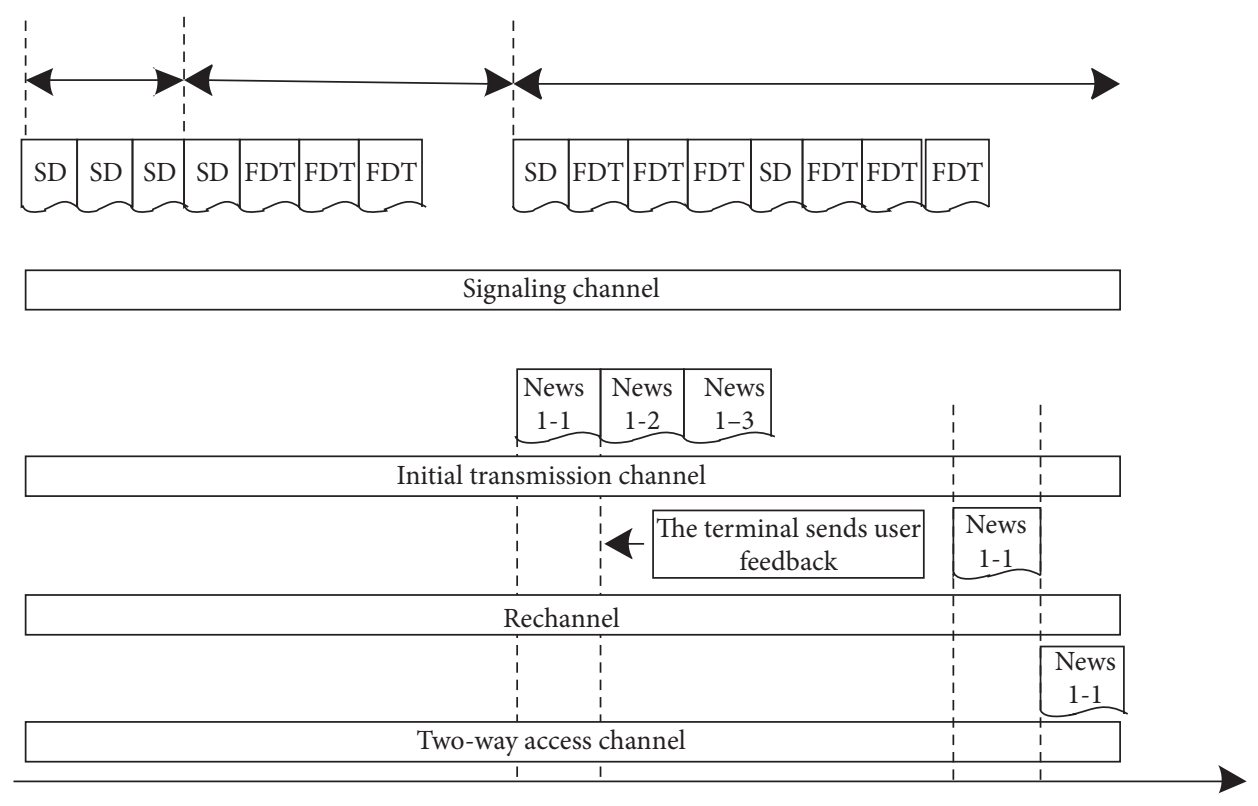

FIGURE 5: Schematic diagram of push server channel arrangement.

initial transmission channel is responsible for the initial input of medical data, the retransmission channel is responsible for sending commands, and the bidirectional access channel provides diversity gain for wireless transmission and improves the reliability of data transmission. If the channel environment is the same, the value is the same, and the channel that meets the restriction conditions and has the shortest push transmission distance can be selected as the push channel of medical data by calculation.

The optimized multidimensional heterogeneous medical data will be pushed through the selected push channel. Before the data push, it is necessary to perform status processing on the sender and the receiver. Afterwards, the corresponding multidimensional heterogeneous medical data are pushed. During the push process, it is necessary to strictly control the data push quality using the controller. It is shown in the following equation:

$$
\begin{aligned}
S_{S} & =\operatorname{MTM}_{\mathrm{ur}} \frac{v \varphi}{2 \omega} \cdot \frac{p\left(d_{\text {sensor }} \mid t n_{o i}\right)}{p\left(e \mid n_{o i}\right)}, \\
S_{S^{\prime}} & =2 v \varphi \cos \omega,
\end{aligned}
$$

where $d_{\text {sensor }}$ represents the channel transmission distance, $e$ represents the control parameter, and $S_{s}$ and $S_{s^{\prime}}$ represent the data quality eigenvalue pushed by the sender and receiver, respectively. When the values of $S_{s}$ and $S_{s^{\prime}}$ are within the interval $[0,1]$, it is determined that the medical data conforms to the push quality and is passed. When the receiver displays the corresponding push message, the intelligent cloud collaborative management system implements the medical data push function.

3.7. Implementation of Multidimensional Heterogeneous Medical Data Push Management. The management of multidimensional heterogeneous medical data push could be implemented after the selection of the above channels, and the push process is described as follows:

Input: original multidimensional heterogeneous medical data set $n$ and channel selection

Output: push results of multidimensional heterogeneous medical data

The intelligent cloud collaborative management system is initialized and multidimensional heterogeneous medical data push is performed. The specific steps are described as follows:

(1) Replacement mapping of multisource heterogeneous data. Multisource heterogeneous data is to expand the main components of homogeneous data. In order to facilitate mathematical calculations, a permutation matrix $P_{m}$ is introduced to conduct permutation mapping on the samples obtained on the cloud server, and the result is recorded as $y$

$$
y=\left(y_{\alpha}, y_{b}\right)^{T}=P_{m} n
$$

The purpose is to gather the same part of the current sample as the isomorphic data in front of the vector, denoted by $y_{a}$, and to place the different part behind the vector, denoted by $y_{b}$.

(2) The sample structure obtained from the sampling survey does not match the overall composition, and this structural difference can be eliminated and restored by weighting. $k$ different objects are randomly selected from the data set $y$ of permutation mapping as the initial clustering center;

(3) The weight of each attribute in class $k$ is initialized to the same value, that is, the weight of any class $C_{k^{\prime}}\left(1 \leq k^{\prime} \leq k\right)$ in attribute $A_{t}(1 \leq t \leq m)$ is $1 / m$; 
(4) The weighted measure of dissimilarity of any object $n_{i} \in y$ and class $C_{i}$ is defined as follows:

$$
D\left(n_{i}, z_{i}\right)=\sum_{i=1}^{N} S_{s}\left(n_{i}^{t}-A_{t}\right)+\sum_{i=1}^{N} S_{s^{\prime}}\left(C_{i}^{t}-A_{t}\right)
$$

According to the above equation, the dissimilarity between the object and the class centers are calculated, and the data object is divided into the class represented by the cluster center closest to it according to the nearest neighbor principle;

(5) The cluster centers are updated. Among them, the numerical attribute part is obtained by calculating the average value of the objects in the same class, and the subtype attribute part is obtained by calculating the fuzzy class center.

The fuzzy class center of the subtype attribute part is expressed as follows:

$$
z_{l}^{c}=\left(z_{l, p+1}^{c}, z_{l, p+2}^{c}, \ldots, z_{l, p+m}^{c}\right)
$$

(6) The weights of each attribute in the numerical and subtype data parts of each class in the fuzzy class center are calculated, so as to update the information source.

The data set of the fuzzy class center conforms to the high-dimensional distribution. Therefore, the cloud data sample weights can be calculated using this high-dimensional distribution, and the calculation expression of the weight $J$ is as follows:

$$
J=\exp \left\{\frac{1}{2}\left|\left(y-z_{l}^{c}\right)^{T}\right| \sum\left(y-z_{l}^{c}\right)^{-1}\right\} .
$$

The weight $J$ calculated according to the equation (18) can be used to update the information source. After the information source is updated, the equation for pushing heterogeneous data is as follows:

$$
\text { push }=J \sum_{i=1}^{N} y_{i}^{T} .
$$

In summary, the multidimensional heterogeneous medical data push is completed, as shown in Figure 6.

\section{Experimental Analysis and Results}

4.1. Experimental Data. In order to verify the application function of the multidimensional heterogeneous medical data push technology in the intelligent cloud collaborative management system, this study sets up an application analysis experiment. The medical data set is selected as follows:

(1) MIMIC Critical Care Database: the public data set from MIT lab for computational physiology collects

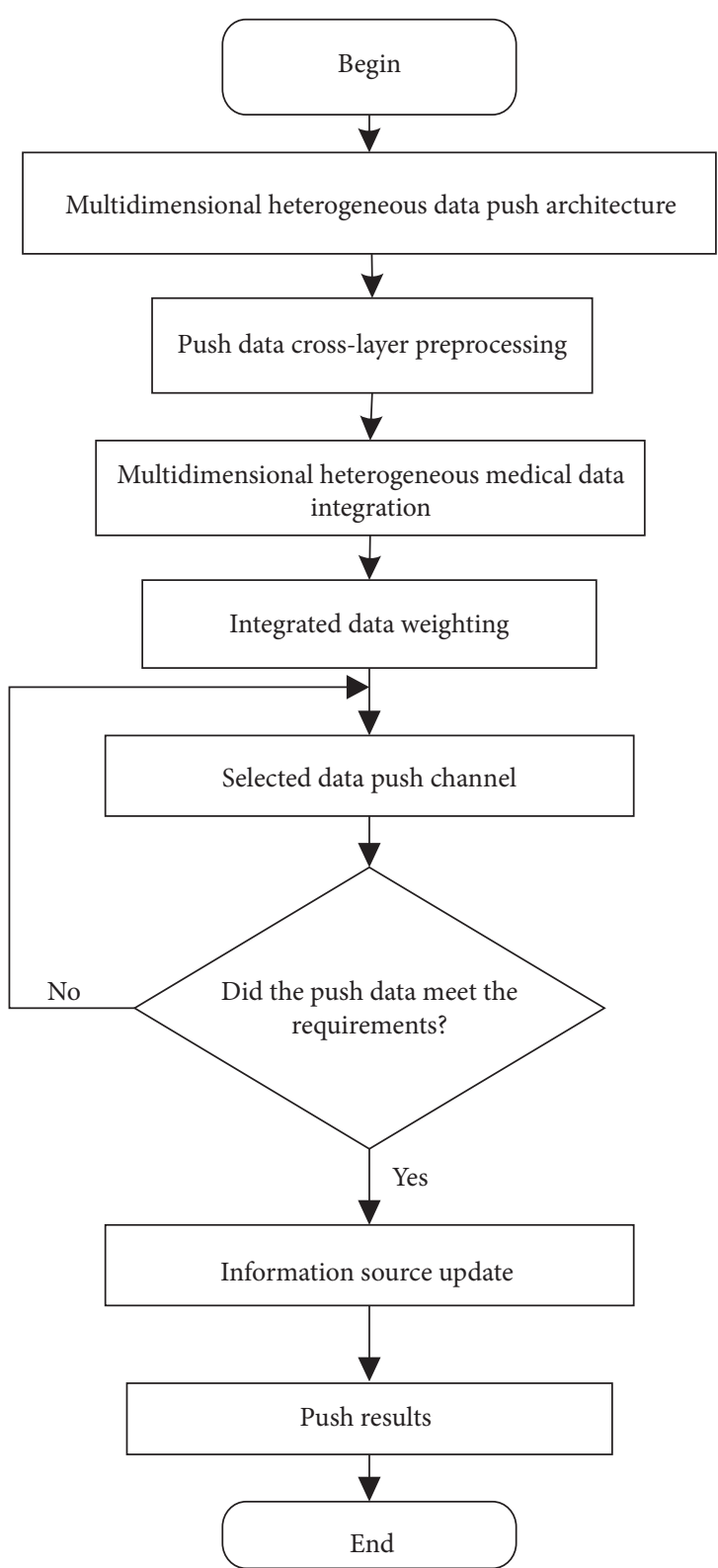

FIgURE 6: Multidimensional heterogeneous medical data push management process.

data from more than 60000 ICUs, including demographic data, physiological signs, laboratory tests, and drug treatment of patients.

(2) Kent Ridge Biomedical Datasets: database in the biomedical field.

4.2. Experimental Steps. This experiment is carried out in a Matlab environment. The GPU model of Inter ${ }^{\circledR}$ CoreTM i54150 is used for cloud collaboration management system training. In this experimental analysis, 10 million data are selected from the above two data sets. The experimental data are processed by the cross-layer preprocessing method in Section 3.3, and effective data are collected and half of the data in each data set is randomly selected as the training 
data, and the rest of the data is used as the test data set for experimental analysis. The two datasets of mimic critical care database and Kent ridge biological datasets are used to further optimize the training process of the cloud collaboration management system. The training cycle is set to 100 cycles and the learning rate is set to 0.001 .

\subsection{Evaluation Criteria}

(1) Data update rate: it refers to the data update rate received by the GPS device, which is generally refreshed in units of 1 time/second. Generally, the data update rate determines how much data an instrument can store, and it also represents the speed of data update. Generally speaking, a faster update rate represents better performance.

(2) Retention rate: it refers to the proportion of users who still retain the pushed message within a certain period of time (such as 1-6 weeks), which can also reflect the impact of the model on users to a certain extent.

(3) Communication rate: it refers to the number of communications between the user and the cloud push platform in a unit time, for testing whether the user is willing to use the platform for data push. The higher the communication rate, the stronger the user's willingness to use the system and the better the system performance.

(4) Precision rate of data push:

$$
\text { precision }=\frac{\mathrm{TP}}{\mathrm{TP}+\mathrm{FP}} \text {. }
$$

where FP is the number of samples that are incorrectly pushed. TP is the number of samples correctly pushed.

(5) Recall rate of data push:

$$
\text { recall }=\frac{\mathrm{TP}}{\mathrm{TP}+\mathrm{FN}},
$$

where $\mathrm{FN}$ is the number of samples incorrectly pushed.

In order to highlight the application value of multidimensional heterogeneous medical data push technology, the architecture method based on $\mathrm{B} / \mathrm{S}$ in literature [16], the approach based on GSM-MBM in literature [17], the approach based on cloud computing in literature [18], the cloud remote collaboration service system in literature [19], the personal health record cloud management system in literature [20], the priority push based on LBS in literature [21], and the Internet-based inpatient health propaganda and education cloud platform in literature [22] were compared with the proposed method, so as to verify the application value of the proposed method.

\subsection{Results and Discussion}

4.4.1. Comparison of the Data Update Rate. In this study, the same update task was assigned to different systems. The update task was divided into 7 stages, each update data volume was $1024 \mathrm{MB}$, and the data update rate of different systems was recorded. It is shown in Figure 7.

According to Figure 7, different collaborative management systems all have higher update rates. After calculation, the average update rates of data for the architecture method based on B/S in literature [16], the approach based on GSMMBM in literature [17], the approach based on cloud computing in literature [18], the cloud remote collaboration service system in literature [19], the personal health record cloud management system in literature [20], the priority push based on LBS in literature [21], and the Internet-based inpatient health propaganda and education cloud platform in literature [22] are $92.075 \%, 91.145 \%, 89.654 \%, 88.567 \%$, $90.521 \%, 70 \%$, and $65 \%$, respectively. Due to the application of the multidimensional heterogeneous medical data push technology, the average update data volume of the intelligent cloud collaborative management system is $1021.125 \mathrm{MB}$, an increase of $77.78 \mathrm{MB}$, and the average update rate is $99.61 \%$, an average increase of $7.535 \%$. Therefore, it can be concluded that the application of multidimensional heterogeneous medical data push technology can effectively increase the data update rate of the intelligent cloud collaborative management system.

4.4.2. Comparison of the Retention Rate. Actually, the retention rate reflects a conversion rate, that is, the process from the initial unstable users into active users, stable users, and loyal users. With the continuous extension of this retention rate statistical process, the changes of users in different periods can be seen. The higher the retention rate, the better the system performance can meet user needs and the better the performance. It is shown in Figure 8 .

According to Figure 8, the average retention rates of the architecture method based on $\mathrm{B} / \mathrm{S}$ in literature [16], the approach based on GSM-MBM in literature [17], the approach based on cloud computing in literature [18], the cloud remote collaboration service system in literature [19], the personal health record cloud management system in literature [20], the priority push based on LBS in literature [21], and the Internet-based inpatient health propaganda and education cloud platform in literature [22] are 6.5\%, $12.5 \%, 10 \%, 12.5 \%, 15 \%, 10 \%$, and $7.5 \%$, respectively. In contrast, the retention rate of the proposed method is basically stable at $20 \%$, and its retention rate is significantly higher than other methods. Therefore, the proposed method has significant advantages.

4.4.3. Comparison of the Communication Rate. The change in the communication rate of different methods is shown in Figure 9. The communication rates of literature [16], literature [17], literature [18], literature [19], literature [20], literature [21], and literature [22] maintain unchanged between $50 \%$ and $65 \%$. In contrast, the communication rate of the proposed method can be as high as about $85 \%$, and finally, tend to stabilize at about $75 \%$ as the time of experiment increases. Therefore, the proposed method is much 


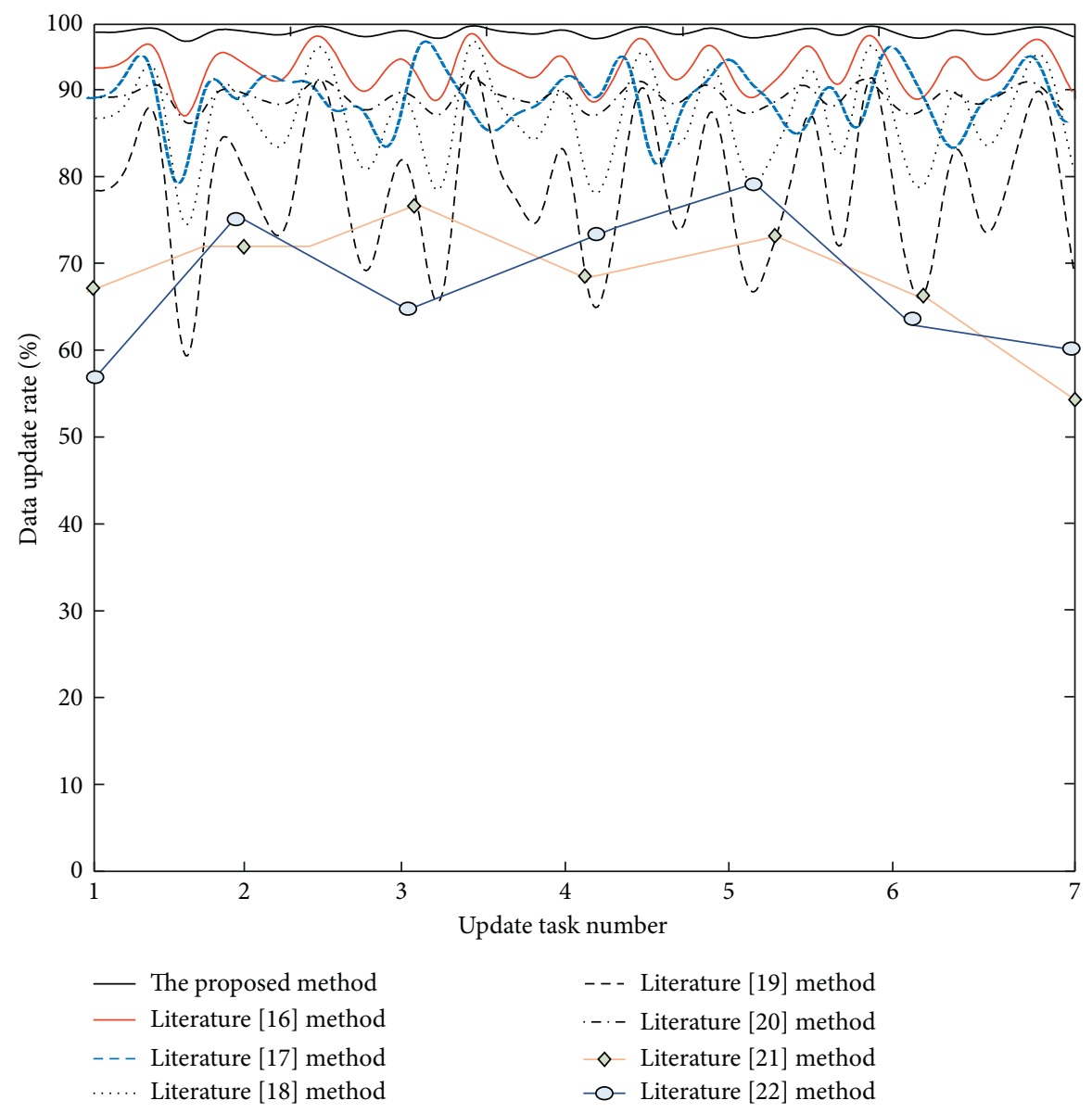

FIgURE 7: Data update rate change results.

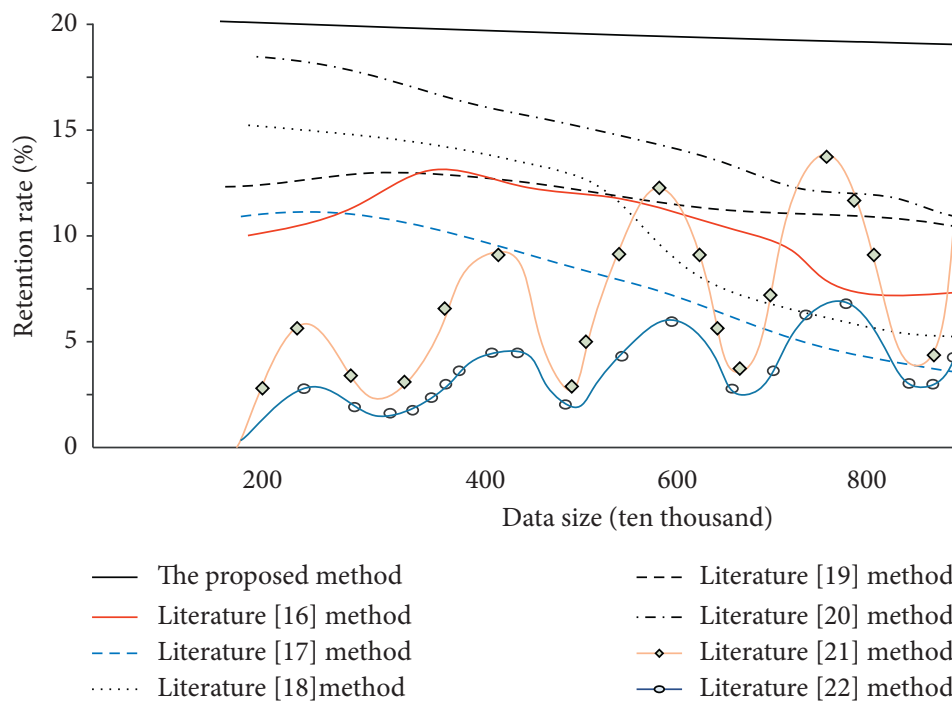

FIGURE 8: Change of retention rate of different methods.

better than other methods. This is mainly because the proposed method selects the push channel in data push, which increases the accuracy of medical data information push and improves the communication rate.
4.4.4. Comparison of the Precision Rate. The precision rate mainly depends on the specificity of the retrieved information and whether the proposed retrieval strategy can accurately express the users' real intelligence needs. It is shown in Figure 10. 


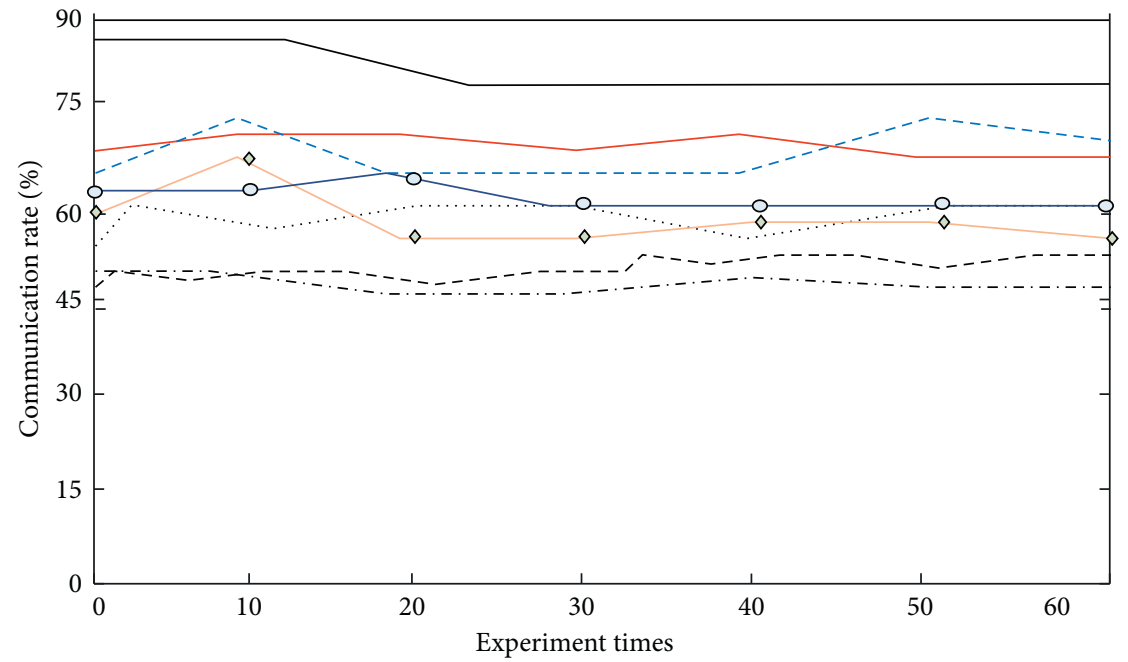
— The proposed method
- - Literature [20] method
- Literature [16] method
$\diamond$ Literature [21] method
- - - Literature [17] method
- - Literature [22] method

- - - Literature [19] method

Figure 9: Change of communication rate of different methods.

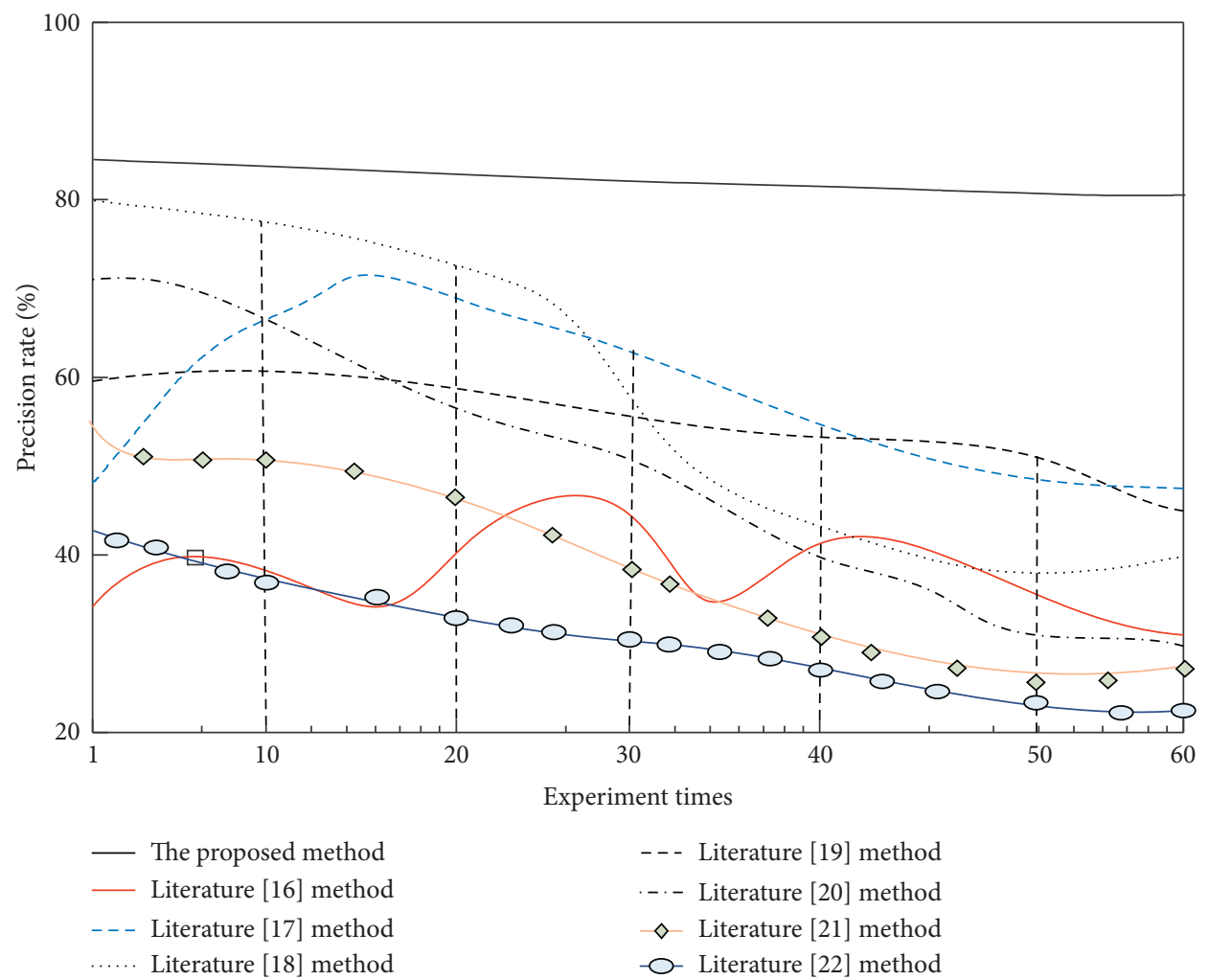

FIGURE 10: Precision rate result.

According to Figure 10, the average precision rates of the architecture method based on $\mathrm{B} / \mathrm{S}$ in literature [16], the approach based on GSM-MBM in literature [17], the approach based on cloud computing in literature [18], the cloud remote collaboration service system in literature [19], the personal health record cloud management system in literature [20], the priority push based on LBS in literature [21], and the Internet-based inpatient health propaganda and education cloud platform in literature [22] are $40 \%$, $60 \%, 60 \%, 58 \%, 35 \%, 30 \%$, and $32 \%$, respectively. In 


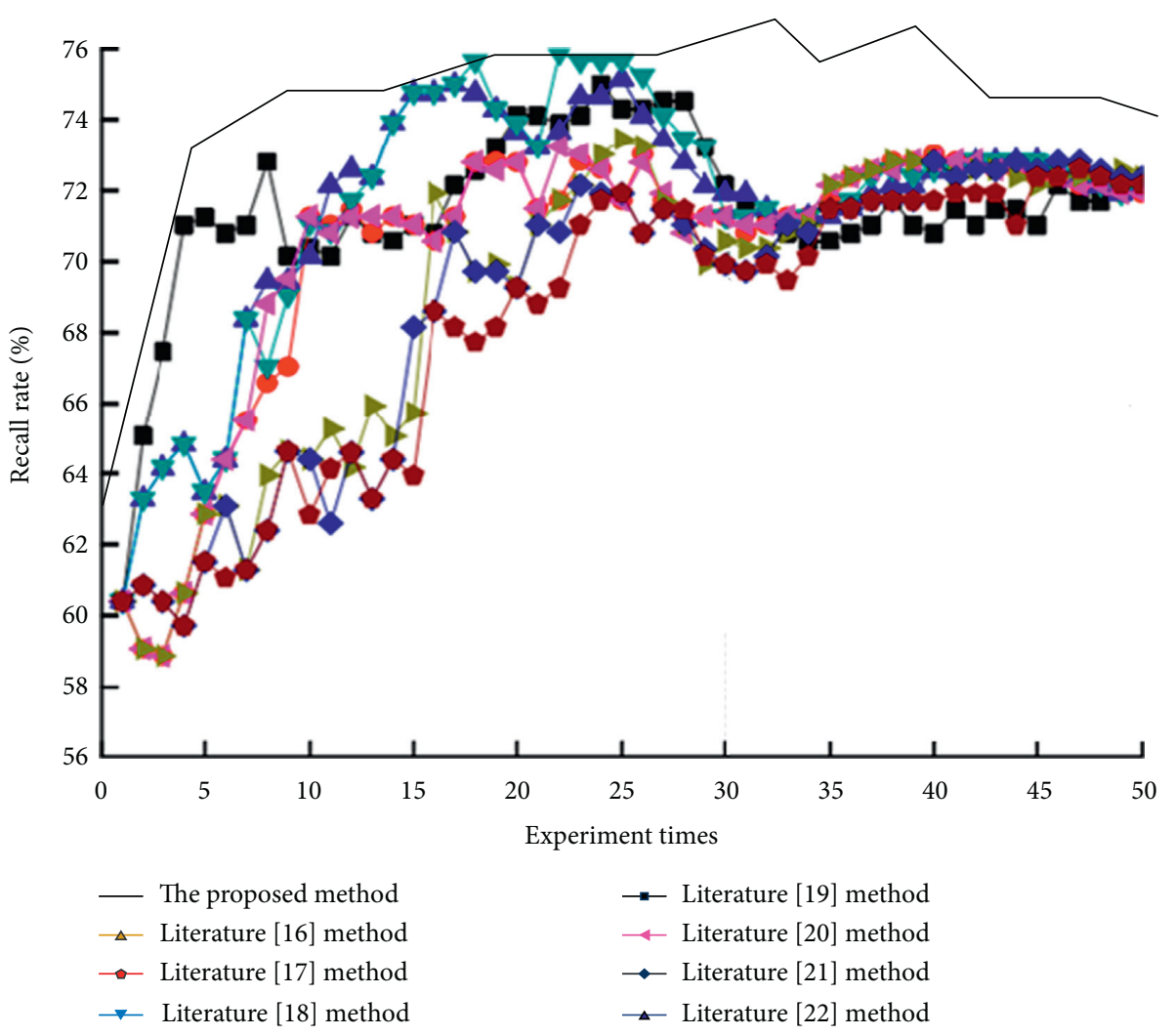

FIgURE 11: Recall rate results.

contrast, the precision rate of the proposed method is more than $80 \%$. The reason is that the proposed method determines the system logical architecture of multidimensional heterogeneous medical data push, makes a weighted analysis of multidimensional heterogeneous medical data, reduces the dimension of medical data, avoids the removal of multidimensional heterogeneous interference, and improves the precision rate.

4.4.5. Comparison of the Recall Rate. The change in recall rate of different methods is shown in Figure 11. The recall rates of the architecture method based on $\mathrm{B} / \mathrm{S}$ in literature [16], the approach based on GSM-MBM in literature [17], the approach based on cloud computing in literature [18], the cloud remote collaboration service system in literature [19], the personal health record cloud management system in literature [20], the priority push based on LBS in literature [21], and the Internet-based inpatient health propaganda, and education cloud platform in literature [22] are not uniform, and their entire experimental process is significantly lower than the proposed method. In contrast, the precision rate of the proposed method is up to $76 \%$. The reason is that the proposed method designs a multidimensional cross-layer data preprocessing method, which enhances the strength of data signals and promotes the improvement of the recall rate.

\section{Conclusions}

The multidimensional heterogeneous medical data is frequently generated in the medical research field, which affects the process of medical data processing. As a result, in-depth research and analysis of multidimensional heterogeneous medical data push is of great benefit to the development of medical systems. This paper analyzes the application of multidimensional heterogeneous medical data push in the intelligent cloud collaborative management system and gives the system logic architecture, and the multidimensional heterogeneous medical data were processed; the push channel was selected, and the data push was effectively completed. The results show that the proposed method has superior performance and good data push performance, which provides a reference basis for the development of the medical field.

However, in actual medical research, due to the diversity of disease types, it is impossible to accurately judge the diseases of many patients, and the accuracy of the results of disease-related data push is affected. Therefore, in further study, we will focus on introducing intelligent systems into disease diagnosis for analysis, providing a data basis for the diagnosis of diverse diseases, helping to obtain more accurate disease diagnosis results, and laying a foundation for further research on the data push system and increasing the intelligence and richness of the system. 


\section{Data Availability}

The data used to support the findings of this study are included within the article. Readers can access the data supporting the conclusions of the study from MIMIC Critical Care Database and Kent Ridge Biomedical Datasets.

\section{Conflicts of Interest}

The authors declare that they have no conflicts of interest.

\section{Acknowledgments}

This work was supported by the Humanities and Social Sciences Research Planning Fund Project in Ministry of Education under grant number 19YJAZH053, the Opening Project of State Key Laboratory of Digital Publishing Technology under grant number cndplab-2020-M003, and Ministry of Education Science and Technology Development Center Industry-University Research Innovation Fund under grant number 2018A01002.

\section{References}

[1] W. Xiao, L. Lu, C. Ji, X. Yu, and D. Qi, "Prediction of water positions in the binding sites of proteins based on collections of multi-source heterogeneous atoms," Chemical Biology \& Drug Design, vol. 95, no. 2, pp. 224-232, 2020.

[2] Y. Guo, Design and Implementation of a Collaborative Management Platform Based on Face Verification, Beijing University of Posts and Telecommunications, Beijing, China, 2018.

[3] P. Sharma, "Prediction of heart disease using 2-tier SVM data mining algorithm," International Journal of Advanced Research in Big Data Management System, vol. 1, no. 2, pp. 11-24, 2017.

[4] J.-H. Bae and H. Y. Lee, "User health information analysis system of urine and feces separable smart toilet," International Journal on Human and Smart Device Interaction, vol. 5, no. 2, pp. 19-24, 2018.

[5] B. Kumwenda, J. A. Cleland, G. J. Prescott, K. Walker, and P. W. Johnston, "Relationship between sociodemographic factors and selection into UK postgraduate medical training programmes: a national cohort study," British Medical Journal Open, vol. 8, no. 6, Article ID e021329, 2018.

[6] A. Care, F. A. Ramponi, M. C. Campi et al., "A new classification algorithm with guaranteed sensitivity and specificity for medical applications," IEEE Control Systems Letters, vol. 2, no. 3, pp. 393-398, 2018.

[7] J. Yu, Z. Kuang, B. Zhang, W. Zhang, D. Lin, and J. Fan, "Leveraging content sensitiveness and user trustworthiness to recommend fine-grained privacy settings for social image sharing," IEEE Transactions on Information Forensics and Security, vol. 13, no. 5, pp. 1317-1332, 2018.

[8] Y. Yuyu, X. Jing, L. Yu, X. Yueshen, X. Wenjian, and Y. Lifeng, "Group-wise itinerary planning in temporary mobile social network,” IEEE Access, vol. 7, pp. 83682-83693, 2019.

[9] Y. Yin, L. Chen, Y. Xu, and J. Wan, "QoS prediction for service recommendation with deep feature learning in edge computing environment," Mobile Networks and Applications, vol. 25, no. 1, pp. 1-11, 2019.

[10] H. Gao, Y. Xu, Y. Yin et al., "Context-aware QoS prediction with neural collaborative filtering for internet-of-things services," IEEE Internet of Things Journal, vol. 7, no. 5, pp. 4532-4542, 2020.

[11] K. Jiang, "Research on feature push of massive medical information based on data feature matrix," Mechanical Design and Manufacturing Engineering, vol. 48, no. 3, pp. 59-63, 2019.

[12] J. Mei, Y. Wang, J. Zhang et al., "Feasibility analysis of the application of information push technology in tuberculosis management of floating population," International Medical and Health Guide, vol. 24, no. 3, pp. 320-323, 2018.

[13] J. Yu, J. Li, Z. Yu, and Q. Huang, "Multimodal transformer with multi-view visual representation for image captioning," IEEE Transactions on Circuits and Systems for Video Technology, vol. 15, p. 1, 2019.

[14] J. Yu, M. Tan, H. Zhang, D. Tao, and Y. Rui, "Hierarchical deep click feature prediction for fine-grained image recognition," IEEE Transactions on Pattern Analysis and Machine Intelligence, 2019, In press.

[15] R. Wang, B. Wu, and L. Yan, "Application of Internet-based inpatient health education cloud platform," International Journal of Nursing, vol. 38, no. 12, pp. 1758-1761, 2019.

[16] L. Bo, "Design and research of hospital electronic medical record management system based on B.Sc. architecture," Electronic Design Engineering, vol. 25, no. 5, pp. 46-49, 2017.

[17] X. Jin, L. Yang, N. Jin et al., "Performance analysis of wireless energy carrying collaboration system based on GSM-MBM," Journal of Beijing University of Posts and Telecommunications, vol. 41, no. 5, pp. 141-146, 2018.

[18] S. Zhang, Y. Luo, and Y. Shen, "Design of Internet of things system for health monitoring of spatial structure based on cloud computing," Spatial Structure, vol. 23, no. 1, pp. 3-11, 2017.

[19] L. Qin, W. Guo, R. Cai et al., "Construction and practice of medical image cloud remote collaboration service system," Biomedical Engineering Research, vol. 27, no. 1, pp. 111-115, 2018.

[20] Q. Liu, X. Liu, B. Hu et al., "Fine-grained access control supporting user revocation in personal health record cloud management system," Journal of Electronics and Information, vol. 39, no. 5, pp. 1206-1212, 2017.

[21] J. B. Cole, S. K. Knack, E. R. Karl, G. B. Horton, R. Satpathy, and B. E. Driver, "Human errors and adverse hemodynamic events related to "push dose pressors" in the emergency department," Journal of Medical Toxicology, vol. 15, no. 4, pp. 276-286, 2019.

[22] A. F. Cartwright, M. Karunaratne, J. Barr-Walker, N. E. Johns, and U. D. Upadhyay, "Identifying national availability of abortion care and distance from major US cities: systematic online search," Journal of Medical Internet Research, vol. 20, no. 5, p. e186, 2018.

[23] B. Fred, "Getting value from EHR data. Analytics push yields payoff at medical center health," Health Data Management, vol. 24, no. 3, pp. 51-53, 2016.

[24] M. Gagolewski, R. Perez-Fernandez, and B. De Baets, "An inherent difficulty in the aggregation of multidimensional data," IEEE Transactions on Fuzzy Systems, vol. 28, no. 3, pp. 602-606, 2020.

[25] M. E. Klijn and J. Hubbuch, "Application of empirical phase diagrams for multidimensional data visualization of highthroughput microbatch crystallization experiments," Journal of Pharmaceutical Sciences, vol. 107, no. 8, pp. 2063-2069, 2018.

[26] S. Ali, Al T. Ahmad, Y. Najwa et al., "Data on the relationship between caffeine addiction and stress among Lebanese 
medical students in Lebanon," Data in Brief, vol. 20, no. 5, p. $104845,2020$.

[27] Y. Jin, X. Guo, Y. Li, J. Xing, and H. Tian, "Towards stabilizing facial landmark detection and tracking via hierarchical filtering: a new method," Journal of the Franklin Institute, vol. 357, no. 5, pp. 3019-3037, 2020.

[28] J. Li, X. Zhang, Z. Wang et al., "Dual-band eight-antenna array design for MIMO applications in 5G mobile terminals," IEEE Access, vol. 7, no. 1, pp. 71636-71644, 2019.

[29] K. Chen, K. S. Dhindsa, and B. Bhushan, "Collaborative agentbased model for distributed defense against DDoS attacks in ISP networks," International Journal of Security and Its Applications, vol. 11, no. 8, pp. 1-12, 2017.

[30] H. Sun and Q. Hu, "A novel deep web data mining algorithm based on multi-agent information system and collaborative correlation rule," International Journal of Future Generation Communication and Networking, vol. 9, no. 11, pp. 81-94, 2016.

[31] E. Zhang, J. Fiaidhi, and S. Mohammed, "Social recommendation using graph database Neo4j: mini blog, twitter social network graph case study," International Journal of Future Generation Communication and Networking, vol. 10, no. 2, pp. 9-20, 2017.

[32] R. Du, Z. Pei, and J. Tian, "Personalized trusted service recommendation method based on social work," International Journal of Security and Its Applications, vol. 10, no. 9, pp. 29-38, 2016.

[33] H. Xi, D. Guo, and H. Zhu, "Application of data mining based on classifier in class label prediction of coal mining data," International Journal of Security and Its Applications, vol. 9, no. 10, pp. 425-432, 2015.

[34] M. Francia, M. Golfarelli, and S. Rizzi, "Summarization and visualization of multi-level and multidimensional itemsets," Information Sciences, vol. 520, pp. 63-85, 2020.

[35] W. Shao, K. Huang, Z. Han et al., "Integrative analysis of pathological images and multidimensional genomic data for early-stage cancer prognosis," IEEE Transactions on Medical Imaging, vol. 39, no. 1, pp. 99-110, 2020. 